\title{
Raoultella ornithinolytica in MALT-type non-Hodgkin Lymphoma
}

\author{
$\underline{\text { Rui Seixas }}{ }^{1}$, Adelaide Alves $^{2}$, Aurelia Selaru ${ }^{3}$, Manuela Vanzeller $^{2}$, Teresa Shiang $^{2}$, Sara Conde $^{2}$ \\ ${ }^{1}$ Internal Medicine Department, Unidade Local de Saúde Litoral Alentejano, Santiago do Cacém, Portugal \\ 2 Pulmonology Department, Centro Hospitalar Vila Nova de Gaia e Espinho, Portugal \\ ${ }^{3}$ Clinical Pathology Department, Centro Hospitalar Vila Nova de Gaia e Espinho, Portugal
}

Received: 22/10/2021

Accepted: 30/10/2021

Published: 16/11/2021

How to cite this article: Seixas R, Alves A Selaru A, Vanzeller M, Shiang T, Conde S. Raoultella ornithinolytica in MALT-type non-Hodgkin lymphoma. EJCRIM 2021;8: doi:10.12890/2021_003023.

Conflicts of Interests: The authors declare there are no competing interests.

This article is licensed under a Commons Attribution Non-Commercial 4.0 License

\section{ABSTRACT}

Raoultella ornithinolytica is a bacterium that belongs to the Enterobacteriaceae family. The most frequently reported infections are gastrointestinal and hepatobiliary. Urinary tract infections are very rarely reported and bloodstream infections are usually reported without an identified source. This bacterium is responsible for an increasing number of infections, especially in immunocompromised patients. The authors describe the first case ever reported of an immunocompromised patient due to non-Hodgkin lymphoma MALT type and corticotherapy, who developed urinary tract infection and subsequently bacteriemia due to this pathogen.

\section{LEARNING POINTS}

- Raoultella ornithinolytica is a virulent pathogen causing community-acquired and hospital-acquired infection, especially in immunocompromised populations.

- $\quad$ Although most cases of $R$. ornithinolytica infection are susceptible to standard antibiotic regimens, multi-drug resistant strains have been reported, which may pose a severe risk to the immunocompromised patient.

- Physicians should be aware that some treatments may increase immunosuppression, thus enabling infection by opportunistic agents such as $R$. ornithinolytica.

\section{KEYWORDS}

Raoultella ornithinolytica, immunosuppression, non-Hodgkin lymphoma

\section{CASE DESCRIPTION}

The patient was a 76-year-old man, previously independent, with a known medical history of non-Hodgkin lymphoma MALT type for which he had already undergone six cycles of R-CHOP chemotherapy (cyclophosphamide, doxorubicin, vincristine, prednisone and rituximab). He had signs of disease progression, chronic renal disease and chronic obstructive pulmonary disease, and was admitted to the Emergency Department due to shortness of breath and prostration with 12 hours of evolution. On admission, he showed clear signs of respiratory distress and respiratory acidosis with $\mathrm{pH} 7.10$, partial pressure of carbon dioxide $\left(\mathrm{pCO}_{2}\right) 107 \mathrm{mmHg}$ and partial pressure of oxygen ( $\mathrm{pO} \mathrm{O}_{2}$ ) $114 \mathrm{mmHg}$. He began immediate treatment with non-invasive mechanical ventilation with clinical improvement. Blood analysis revealed a slight elevation in C-reactive protein (CRP) to $5.4 \mathrm{mg} / \mathrm{dl}$. Blood and urine cultures were obtained but no pathogens were identified. Chest $\mathrm{x}$-ray showed bilateral diffuse infiltrates and chest tomography revealed a nodule in the lower lobe of the right lung. Review of the patient's medical history showed that previous CT scans had revealed this nodule and that transthoracic biopsy had excluded pulmonary infiltration by the non-Hodgkin lymphoma. 
The patient was transferred to an Intermediate Care Unit in the Internal Medicine Department, diagnosed with probable usual interstitial pneumonia, and began treatment with methylprednisolone pulses ( $1 \mathrm{~g}$ per day) for 5 days. The patient showed clinical improvement and was transferred to the Pulmonology Department where he maintained corticotherapy with prednisolone 60 mg daily. After 3 days, the patient presented fever (temperature $38.3^{\circ} \mathrm{C}$ ) and significantly elevated CRP (12 mg/dl; normal <0.5 mg/dl) and procalcitonin (15 ng/ml; normal $0.5 \mathrm{ng} / \mathrm{ml}$ ). Urine culture and two sets of blood cultures all identified $R$. ornithinolytica resistant to amoxicillin but sensitive to cotrimoxazole (Table 1). The patient began treatment with cotrimoxazole with resolution of a urinary tract infection (UTI) and bloodstream infection. He showed progressive clinical improvement and responded well to weaning from corticotherapy. His hospitalization was prolonged due to acalculous acute pancreatitis but he was discharged 3 weeks later.

\begin{tabular}{|c|c|c|}
\hline \multicolumn{3}{|c|}{ Microbiological study } \\
\hline Blood cultures (both sets) & \multicolumn{2}{|c|}{ Antibiotic susceptibility testing } \\
\hline \multirow{7}{*}{$\begin{array}{c}\text { Pathogen: } \\
\text { Raoultella ornithinolytica }\end{array}$} & Amoxicillin & Resistant \\
\hline & Amoxicillin/clavulanic acid & Susceptible \\
\hline & Cefotaxime & Susceptible \\
\hline & Ceftriaxone & Susceptible \\
\hline & Cotrimoxazole & Susceptible \\
\hline & Cefuroxime & Resistant \\
\hline & Gentamicin & Susceptible \\
\hline Urine culture & \multicolumn{2}{|c|}{ Antibiotic susceptibility testing } \\
\hline \multirow{6}{*}{$\begin{array}{c}\text { Pathogen: } \\
\text { Raoultella ornithinolytica }\end{array}$} & Amoxicillin & Resistant \\
\hline & Amoxicillin/clavulanic acid & Susceptible \\
\hline & Cefotaxime & Susceptible \\
\hline & Cotrimoxazole & Susceptible \\
\hline & Cefuroxime & Resistant \\
\hline & Gentamicin & Susceptible \\
\hline
\end{tabular}

Table 1. Microbiological study and antibiotic susceptibility testing

\section{DISCUSSION}

Raoultella ornithinolytica is a gram-negative, capsulated, aerobic and non-motile bacterium belonging to the Enterobacteriaceae family. Although previously classified as Klebsiella, this bacterium now belongs to a new genus Raoultella, which includes three different species: $R$. ornithinolytica, $R$. planticola and $R$. terrigen ${ }^{[1]}$. This bacterium has been found predominantly in water environments but also in soil, fish and insects ${ }^{[2]}$. The incidence of human disease associated with $R$. ornithinolytica is low, with few cases requiring antibiotic regimens. This might be due to the difficulty of properly identifying this bacterium with most phenotypic and biochemical tests due to its phenotypic similarity 
to Klebsiella species ${ }^{[3]}$. However, the number of infections requiring treatment has recently increased and some multi-drug resistant strains have been reported ${ }^{[1]}$. Although $R$. ornithinolytica causes gastrointestinal and hepatobiliary infection, it can also affect other organs, such as the urinary tract, but far fewer such cases have been reported. There have been some cases of systemic infection with R. ornithinolytica, presenting mainly as bacteraemia, but without an identifiable source for the infection. Notably, many of the reported cases were in patients with either immunodeficiencies or malignant conditions, making them more complex infections and not just community acquired ${ }^{[1]}$. Infections by this bacterium are still unusual in cancer patients, although there have been rare cases of UTI [4]. Very few cases of infection with $R$. ornithinolytica have been reported in patients with lymphomas and even fewer in patients with non-Hodgkin lymphoma, with only three cases described so far. To the best of our knowledge, this is the first time a UTI with progression to a bloodstream infection by $R$. ornithinolytica has been reported in a patient with non-Hodgkin lymphoma MALT type. The changes these lymphomas can cause in the immune systems of patients may allow opportunistic bacteria such as R. ornithinolytica to produce infection. This patient was immunosuppressed because of his cancer, while the high-dose corticotherapy further compromised his immune system, thus allowing this bacterium to provoke systemic infection. Although the antibiotic susceptibility tests showed very little resistance, the authors emphasize that multi-drug resistant strains of this pathogen have been reported and so the infectious potential of this bacterium is increasingly relevant. $R$. ornithinolytica is a virulent pathogen, although very rare in the clinical setting, and when identified as the cause of infection, especially bacteriemia, should always be treated and antimicrobial susceptibility tests performed.

\section{REFERENCES}

1. Hajjar R, Ambaraghassi G, Sebajang H, Schwenter F, Su SH. Raoultella ornithinolytica: emergence and resistance. Infect Drug Resist 2020;13:1091-1104. doi: 10.2147/IDR. S191387.

2. Levorova J, Machon V, Guha A, Foltan R. Septic arthritis of the temporomandibular joint caused by rare bacteria Raoultella ornithinolytica. Int J Oral Maxillofac Surg 2017;46(1):111-115. doi: 10.1016/j.ijom.2016.09.008. PMID: 27726907.

3. Chun S, Yun JW, Huh HJ, Lee NY. Clinical characteristics of Raoultella ornithinolytica bacteremia. Infection 2015;43(1):59-64. doi: 10.1007/s15010-014-0696-z. PMID: 25367410 .

4. García-Lozano T, Pascual Plá FJ, Aznar Oroval E. Raoultella ornithinolytica en infecciones de las vías urinarias. Estudio clínico y microbiológico de una serie de 4 pacientes con neoplasias [Raoultella ornithinolytica in urinary tract infections. Clinical and microbiological study of a series of 4 oncologic patients] [In Spanish]. Med Clin (Barc) 2013;141(3):138-139. doi: 10.1016/j.medcli.2012.11.021. PMID: 23332629 